J. Phys. IV France 127 (2005) 39-42

(C) EDP Sciences, Les Ulis

DOI: 10.1051/jp4:2005127007

\title{
Décharges luminescentes impulsionnelles et sans mercure
}

\author{
E. Robert, C. Cachoncinlle, R. Viladrosa, S. Dozias, S. Point \\ et J.-M. Pouvesle
}

GREMI, CNRS-Polytech'Orléans, 14 rue d'Issoudun, BP. 6744, 45067 Orléans Cedex 2, France

\begin{abstract}
Résumé. Ce papier présente les études réalisées au GREMI en collaboration avec la société AUPEM SEFLI pour le développement d'enseignes lumineuses sans mercure. Les travaux concernent la caractérisation électrique, spectroscopique et photométrique de décharges luminescentes à base de gaz rares (notamment néon et xénon) produites par un générateur compact d'impulsions électriques. Au-delà, de l'augmentation très significative du flux lumineux et de l'efficacité énergétique en comparaison avec les performances obtenues par l'emploi de ballasts conventionnels, la spectroscopie résolue en temps des plasmas de colonne positive et des régions proches des électrodes permet d'appréhender les conditions de fonctionnement des enseignes sans mercure et la cinétique réactionnelle pendant et après (régime de post décharge) l'application des impulsions électriques.
\end{abstract}

\section{CONTEXTE}

Cette étude s'inscrit dans le cadre des recherches dédiées à la suppression du mercure des dispositifs d'éclairage et de signalisation de type fluorescent. Un premier programme de recherche initié par AUPEM SEFLI, société spécialisée dans la production des composants nécessaires à la réalisation d'enseignes (tubes et alimentations), en collaboration avec le CPAT de Toulouse avait permis de déterminer les mélanges à base de gaz rares les plus favorables au fonctionnement d'enseignes lumineuses. L'étude des propriétés de la décharge dans la région proche des électrodes [1] (chute cathodique, coefficient d'émission secondaire, etc.) et l'optimisation du peuplement des raies de résonance du xénon $(129,5$ et $147 \mathrm{~nm}$ ) dans la colonne positive [2] ont permis d'obtenir des systèmes présentant une durée de vie comparable à celle des dispositifs mercurés ( $\sim 15000$ heures) mais délivrant un flux lumineux visible de 5 à 10 fois inférieur. Depuis nos travaux s'orientent vers l'étude des propriétés des enseignes à base de gaz rares alimentées par des impulsions microseconde [3].

\section{DISPOSITIF EXPÉRIMENTAL}

Ce papier présente des résultats obtenus sur des enseignes lumineuses scellées renfermant soit un mélange de néon et de xénon soit un mélange à trois composants (néon, hélium et xénon). Ce tube, en série avec une résistance de mesure, est alimenté par un générateur d'impulsions électriques. Des sondes de tensions enregistrent l'allure temporelle de l'impulsion de tension entre les électrodes ainsi que l'évolution temporelle du courant parcourant le tube. Ces mesures électriques permettent de connaître la puissance électrique délivrée au tube, la puissance globale consommée (tube et alimentation) est mesurée par un compteur relié au secteur.

Les mesures de spectroscopie sont ici réalisées au travers de la paroi du tube en verre (borosilicate) soit dans la colonne positive soit dans la région située en sortie des électrodes. Le tube est placé devant un spectromètre Acton Research Company VM 504 équipé de deux réseaux (300 et 1200 traits/mm) couplé soit à un tube photomultiplicateur soit à une caméra CCD intensifiée (PI max, Princeton Instruments). Les enseignes lumineuses fonctionnent dans le régime de décharge luminescente dans un milieu à basse pression (qq. mbar). La grande durée de vie des systèmes et l'efficacité optimale sont obtenues par l'emploi 
d'électrodes creuses et froides pour lesquelles l'émission secondaire permet l'entretien de la décharge. La décharge est ainsi créée sous haute tension (domaine $\mathrm{kV}$ ) et des courants moyens de quelques dizaines de mA. Les ballasts conventionnels fonctionnent soit à $50 \mathrm{~Hz}$ soit à des fréquences d'environ $25 \mathrm{kHz}$.

Un générateur compact $\left(7 \mathrm{dm}^{3}\right)$ développé au laboratoire délivre des impulsions de largeur temporelle variable (domaine microseconde), d'amplitude ajustable à une fréquence de répétition également variable (domaine kilohertz). Le système permet l'allumage de tubes de plusieurs dizaines de $\mathrm{cm}$ de longueur pour des diamètres intérieurs classiquement utilisés (13 ou $18 \mathrm{~mm}$ ). Pour respecter les conditions nécessaires à un fonctionnement sur plusieurs milliers d'heures, chacune des électrodes est alimentée successivement par des impulsions de même amplitude mais de polarités opposées.

\section{RÉSULTATS}

La figure 1 présente l'évolution de l'illuminance d'une enseigne sans mercure ( $\mathrm{He} / \mathrm{Ne} / \mathrm{Xe})$ en fonction de la puissance électrique injectée en régime alternatif et impulsionnel.

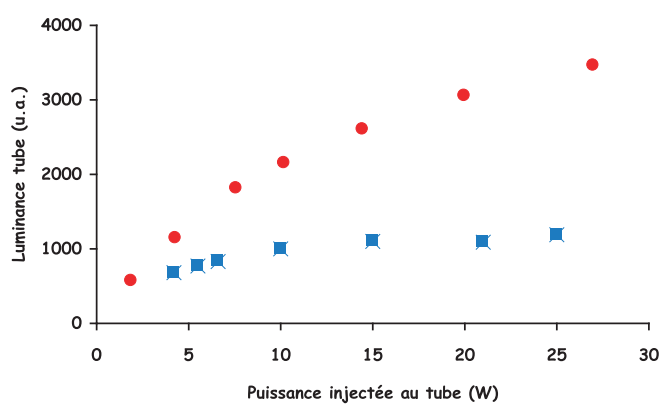

Figure 1. Evolution de l'illuminance d'une enseigne $\mathrm{He} / \mathrm{Ne} / \mathrm{Xe}$ en fonction de la puissance électrique en régime d'excitation alternatif (carré) et impulsionnel (points).

Tandis que dans le cas de l'excitation conventionnelle, le flux visible recueilli présente une saturation pour des puissances supérieures à une dizaine de watt, l'excitation par des impulsions électriques permet d'obtenir des performances trois à quatre fois supérieures. Ainsi à la fois le flux lumineux mais également l'efficacité énergétique apparaissent bien supérieurs dans le cas d'une excitation impulsionnelle pour des dispositifs fluorescents sans mercure. Nous avons aussi mesuré que dans le cas d'une excitation impulsionnelle, l'illuminance des tubes sans mercure est principalement fonction de la puissance délivrée au tube et ne dépend que plus faiblement des conditions (largeur, amplitude et fréquence) électriques qui permettent de délivrer cette puissance.

La figure 2 présente l'évolution temporelle de la fluorescence d'un phosphore bleu (phosphore à rémanence courte) pour deux conditions (couple largeur d'impulsion - fréquence) différentes. Dans les deux cas la fluorescence est produite pendant l'impulsion de courant et dans la phase de post décharge. On note cependant que le rapport d'amplitude de fluorescence pendant les deux phases (décharge et post décharge) est sensible aux conditions d'excitation. De plus, le plasma produit lors des deux phases est différent ce qui conduit à une variation des coordonnées chromatiques du tube en fonction des paramètres de l'alimentation impulsionnelle.

L'optimum de rendement, rapport du flux visible produit à la puissance électrique consommée, est obtenu en ajustant la largeur et la fréquence de répétition des impulsions. Ce réglage des paramètres d'excitation va de paire avec une modification très nette du profil temporel des impulsions électriques. Le générateur de tension est effectivement relié au tube, c'est-à-dire à une impédance variable au cours de l'impulsion et durant la post décharge. L'augmentation de la fréquence conduit à appliquer les impulsions non plus au mélange de gaz neutre mais à un plasma au cours de sa phase de relaxation (post décharge). La phase de décharge a été analysée pour étudier l'allumage des tubes en régime impulsionnel. La 


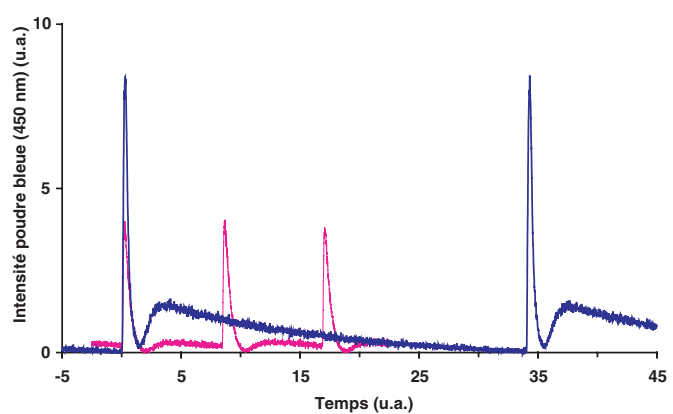

Figure 2. Evolution temporelle de l'émission d'un phosphore produite par une décharge dans un mélange Ne/Xe dans deux conditions d'excitation distinctes : trait gras foncé (largeur temporelle grande et fréquence faible) et trait fin clair (largeur temporelle plus courte et fréquence plus élevée).

figure 3 présente les enregistrements du courant, de la tension ainsi que l'évolution temporelle de trois raies représentatives des espèces présentes dans la zone proche des électrodes dans une enseigne à base de néon et de xénon excitée en régime d'impulsions.

Il apparaît ainsi que le premier pic de courant, enregistré environ 400 ns après l'application de l'impulsion de tension aux bornes du tube, correspond au peuplement d'états excités du néon principalement dans la zone proche des électrodes. Pour les longueurs de tubes étudiées (environ un mètre), cette première phase de la décharge conduit à l'allumage du tube. La montée de l'intensité du courant au cours de l'impulsion est ensuite plus douce. Dans cette deuxième phase, comprise entre 0,5 et

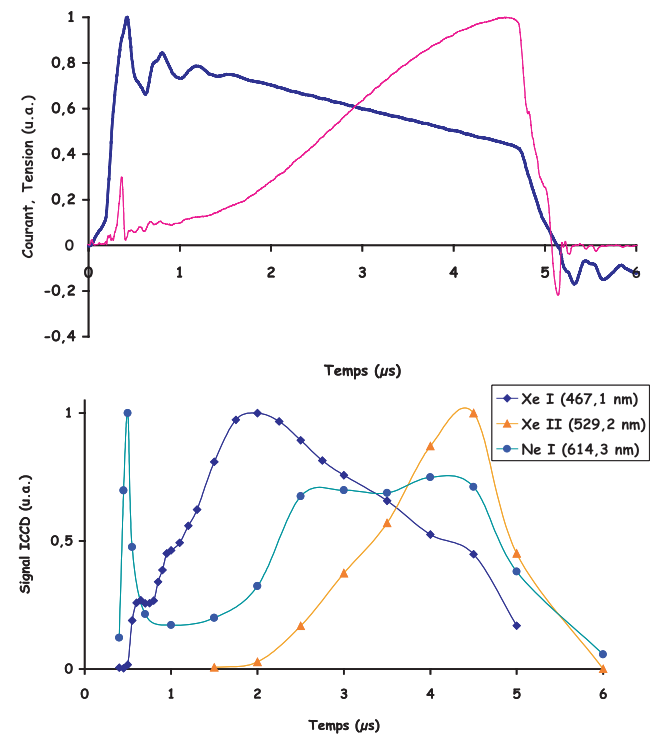

Figure 3. Cinétique de la décharge.

Graphe du haut : allures temporelles des impulsions de tension (trait gras sombre) et du courant (trait fin clair) aux bornes d'une enseigne à base de néon et xénon.

Graphe du bas : profils temporels des raies Ne I $\left(3 \mathrm{~s}\left[1^{1} / 2\right]^{\circ}-3 \mathrm{p}\left[1^{1} / 2\right], 16,62-18,63 \mathrm{eV}\right.$, cercles), Xe I $\left(6 \mathrm{~s}\left[1^{1} / 2\right]^{\circ}\right.$ $7 \mathrm{p}\left[2^{1} / 2\right], 8,31-10,97 \mathrm{eV}$, losanges) et Xe II $\left(6 \mathrm{~s}^{4} \mathrm{P}-6 \mathrm{p}^{4} \mathrm{P}^{\circ}, 23,67-26,02 \mathrm{eV}\right.$, triangles) mesurés pour ces conditions (tension et courant) d'excitation. Les profils temporels des trois raies ont été obtenus en intégrant la lumière du plasma durant 50 ns à différents instants au cours du pulse de courant à l'aide de la caméra ICCD. 
$2 \mu$ s environ, les états supérieurs des transitions du néon se dépeuplent tandis que les transitions des états excités du xénon neutres atteignent leur maximum d'intensité environ deux microsecondes après le début de l'impulsion de tension. La dernière phase de l'impulsion de courant s'établit ensuite. Au même instant, on enregistre une diminution des transitions du xénon neutre, un repeuplement des états supérieurs des transitions du néon et l'apparition des transitions radiatives de l'ion de xénon une fois chargé (XeII). Dans nos conditions expérimentales (la coupure basse en longueur d'onde du verre borosilicate se situe vers $350 \mathrm{~nm}$ ), un ensemble de transitions de l'ion xénon est mesuré entre 480 et $550 \mathrm{~nm}$. Ces raies sont issues de niveaux d'énergie supérieurs situés aux environs de $26 \mathrm{eV}$. Le peuplement de ces états et les profils temporels décrits par la figure 3 , traduisent la cinétique réactionnelle au sein du plasma. Le peuplement des états excités des ions de xénon résulte a priori en grande partie de processus impliquant les états excités et les ions du néon et du xénon et non d'un peuplement direct par excitation électronique.

\section{CONCLUSION ET PERSPECTIVES}

Le développement et l'utilisation d'un générateur compact d'impulsions à paramètres (largeur, amplitude et fréquence de répétition des impulsions) ajustables ont permis d'augmenter de plus d'un facteur trois le flux lumineux visible produit par des enseignes à base de gaz rares sans mercure. L'illuminance des tubes est essentiellement fonction de la puissance électrique injectée à la décharge. Cependant, l'existence d'une phase de post décharge en régime d'excitation impulsionnel permet un repeuplement efficace des états excités du xénon dont les raies de résonance VUV (129,5 et $147 \mathrm{~nm}$ ) qui produisent l'excitation des poudres phosphorescente utilisées en éclairage publicitaire. L'ajustement des paramètres du générateur électrique permet de contrôler le flux lumineux mais également les coordonnées chromatiques de l'enseigne. Une étude cinétique durant la phase de décharge dans les zones proches des électrodes permet l'analyse de l'allumage des plasmas en régime impulsionnel. Des études cinétiques complémentaires, notamment dans le domaine UV-VUV (110-300 nm) et résolues dans l'espace, sont en cours pour fournir une description plus détaillée de la dynamique du plasma dans les décharges sans mercure excitées en régime impulsionnel. Des études de vieillissement, les durées de vie visées étant de plusieurs milliers d'heures, sont également menées sur des enseignes lumineuses sans mercure allumées par un générateur impulsionnel. Il faut rappeler qu'une des problématiques actuelles des dispositifs d'éclairage fluorescent à base de gaz rares tient dans la disparition du xénon du mélange gazeux au cours du temps [3,4]. Cette disparition peut se produire sur des temps très courts, quelques heures, mais il a aussi été démontré la possibilité d'obtenir un fonctionnement correct d'enseignes lumineuses à base de gaz rares sur une durée d'environ 15000 heures à l'aide de ballasts conventionnels [3]. L'étude de l'influence du mode d'excitation sur la modification du mélange gazeux au cours du temps apparaît ainsi cruciale pour le développement de futures enseignes lumineuses écologiques.

\section{Remerciements}

Ces travaux sont financés par la société AUPEM SEFLI, rue Lavoisier, 45504 Gien Cedex. Les auteurs remercient J. Gayet pour le développement des alimentations impulsionnelles.

\section{Bibliographie}

[1] H. Capdeville, Ph. Guillot, L.C. Pitchford, J. Galy, H. Brunet, proc. XXIV ICPIG, vol. III, 5, 1999.

[2] H. Sarroukh, E. Robert, C. Cachoncinlle, R. Viladrosa, J.M. Pouvesle, proc. $9^{\text {th }}$ Intl Symp. on the Science and Technol. of Light Sources, 353, 2001.

[3] E. Robert, H. Sarroukh, C. Cachoncinlle, R. Viladrosa, V. Hochet, S. Eddaoui, J.M. Pouvesle, accepté à paraître dans Pure and Applied Chemistry (2004).

[4] D. Uhrlandt, R. Bussiahn, S. Gortchakov, H. Lange, D. Loffhagen, proc. $11^{\text {th }}$ Intl Symp. on the Science and Technol. of Light Sources, 15, 2004. 